\title{
INTUITIONISTIC FUZZY SEMI-GENERALIZED IRRESOLUTE MAPPINGS
}

\author{
R. SANTHI and K. ARUN PRAKASH
}

\begin{abstract}
The purpose of this paper is to introduce and study the concepts of intuitionistic fuzzy semi-generalized continuous mappings and intuitionistic fuzzy semi-generalized irresolute mappings in intuitionistic fuzzy topological space.
\end{abstract}

\section{Introduction}

The concept of fuzzy set was introduced by Zadeh in his classical paper [14] in 1965. Using the concept of fuzzy sets, Chang [3] introduced the concept of fuzzy topological space. In [1], Atanassov introduced the notion of intuitionistic fuzzy sets in 1986. Using the notion of intuitionistic fuzzy sets, Coker [4] defined the notion of intuitionistic fuzzy topological spaces in 1997. This approach provided a wide field for investigation in the area of fuzzy topology and its applications. One of the directions is related to the properties of intuitionistic fuzzy sets introduced by Gurcay [6] in 1997.

Continuing the work done in the paper [12], we define the notion of intuitionistic fuzzy semi-generalized continuous mappings and intuitionistic fuzzy semi-generalized irresolute mappings. We discuss characterizations of intuitionistic fuzzy semi-generalized continuous mappings and irresolute mappings. We also established their properties and relationships with other classes of early defined forms of intuitionistic continuous mappings.

\section{Preliminaries}

Definition 2.1 ([1]). An intuitionistic fuzzy set (IFS, for short) $A$ in $X$ is an object having the form

$$
A=\left\{\left\langle x, \mu_{A}(x), \gamma_{A}(x)\right\rangle \mid x \in X\right\}
$$

where the functions $\mu_{A}: X \rightarrow[0,1]$ and $\gamma_{A}: X \rightarrow[0,1]$ denote the degree of the membership (namely $\mu_{A}(x)$ ) and the degree of non- membership (namely $\gamma_{A}(x)$ ) of each element $x \in X$ to the set $A$, respectively, $0 \leq \mu_{A}(x)+\gamma_{A}(x) \leq 1$ for each $x \in X$.

Corresponding author: R. Santhi.

2000 Mathematics Subject Classification. 54A40, 03 F55.

Key words and phrases. Intuitionistic fuzzy topology, intuitionistic fuzzy semi-generalized closed set, intuitionistic fuzzy semi-generalized continuous, intuitionistic fuzzy semi-generalized irresolute. 
Definition 2.2 ([1]). Let $A$ and $B$ be IFS's of the forms $A=\left\{\left\langle x, \mu_{A}(x), \gamma_{A}(x)\right\rangle \mid x \in X\right\}$ and $B=$ $\left\{\left\langle x, \mu_{B}(x), \gamma_{B}(x)\right\rangle \mid x \in X\right\}$. Then,

(a) $A \leq B$ if and only if $\mu_{A}(x) \leq \mu_{B}(x)$ and $\gamma_{A}(x) \geq \gamma_{B}(x)$ for all $x \in X$,

(b) $A=B$ if and only if $A \leq B$ and $B \leq A$,

(c) $\bar{A}=\left\{\left\langle x, \gamma_{A}(x), \mu_{A}(x)\right\rangle \mid x \in X\right\}$,

(d) $A \cap B=\left\{\left\langle x, \mu_{A}(x) \wedge \mu_{B}(x), \gamma_{A}(x) \vee \gamma_{B}(x)\right| x \in X\right\}$,

(e) $A \cup B=\left\{\left\langle x, \mu_{A}(x) \vee \mu_{B}(x), \gamma_{A}(x) \wedge \gamma_{B}(x)\right| x \in X\right\}$,

(f) $0_{\sim}=\{\langle x, 0,1\rangle, x \in X\}$ and $1_{\sim}=\{\langle x, 1,0\rangle, x \in X\}$,

(g) $\overline{\bar{A}}=A, \overline{1_{\sim}}=0 \sim$ and $\overline{0_{\sim}}=1_{\sim}$.

Definition 2.3 ([1]). Let $\alpha, \beta \in[0,1]$ with $\alpha+\beta \leq 1$. An intuitionistic fuzzy point (IFP), written as $p_{(\alpha, \beta)}$, is defined to be an IFS of $X$ given by

$$
p_{(\alpha, \beta)}(x)= \begin{cases}(\alpha, \beta), & \text { if } x=p, \\ (0,1), & \text { otherwise. }\end{cases}
$$

Definition 2.4 ([4]). An intuitionistic fuzzy topology (IFT for short) on $X$ is a family $\tau$ of IFS's in $X$ satisfying the following axioms:

(i) $0_{\sim}, 1_{\sim} \in \tau$,

(ii) $G_{1} \cap G_{2} \in \tau$ for any $G_{1}, G_{2} \in \tau$,

(iii) $\cup G_{i} \in \tau$ for any family $\left\{G_{i} \mid i \in J\right\} \subseteq \tau$.

In this case the pair $(X, \tau)$ is called an intuitionistic fuzzy topological space (IFTS for short) and any IFS in $\tau$ is known as an intuitionistic fuzzy open set (IFOS for short) in $X$. The complement $\bar{A}$ of an IFOS A in $\operatorname{IFTS}(X, \tau)$ is called an intuitionistic fuzzy closed set (IFCS for short) in $X$.

Definition 2.5 ([1]). Let $f$ be a mapping from a set $X$ to a set $Y$. If

$$
B=\left\{\left\langle y, \mu_{B}(y), \gamma_{B}(y)\right\rangle ; y \in Y\right\}
$$

is an IFS in $Y$, then the preimage of $B$ under $f$, denoted by $f^{-1}(B)$, is the IFS in $X$ defined by

$$
f^{-1}(B)=\left\{\left\langle x, f^{-1}\left(\mu_{B}(x)\right), f^{-1}\left(\gamma_{B}(x)\right)\right\rangle ; x \in X\right\} .
$$

Definition 2.6 ([4]). Let $(X, \tau)$ be an IFTS and $A=\left\langle x, \mu_{A}, \gamma_{A}\right\rangle$ be an IFS in $X$. Then the intuitionistic fuzzy interior and intuitionistic fuzzy closure of $A$ are defined by

$$
\operatorname{int}(A)=\cup\{G \mid G \text { is an IFOS in } X \text { and } G \subseteq A\},
$$




$$
\operatorname{cl}(A)=\cap\{K \mid K \text { is an IFCS in } X \text { and } A \subseteq K\} .
$$

Note that, for any IFS $A$ in $(X, \tau)$, we have

$$
\operatorname{cl}(\bar{A})=\overline{\operatorname{int}(A)} \quad \text { and } \quad \operatorname{int}(\bar{A})=\overline{\operatorname{cl}(A)} .
$$

Definition 2.7. An IFS $A=\left\{\left\langle x, \mu_{A}(x), \gamma_{A}(x)\right\rangle \mid x \in X\right\}$ in an IFTS $(X, \tau)$ is called

(i) intuitionistic fuzzy semi open set (IFSOS) if $A \subseteq \operatorname{cl}(\operatorname{int}(A))$. [6]

(ii) intuitionistic fuzzy $\alpha$-open set (IF $\alpha \mathrm{OS})$ if $A \subseteq \operatorname{int}(\operatorname{cl}(\operatorname{int}(A)))$.[6]

(iii) intuitionistic fuzzy preopen set (IFPOS) if $A \subseteq \operatorname{int}(\operatorname{cl}(A))$.[6]

(iv) intuitionistic fuzzy regular open set (IFROS) if int $(\operatorname{cl}(A))=A$.[6]

(v) intuitionistic fuzzy semi-pre open set (IFSPOS) if there exists $B \in \operatorname{IFPO}(X)$ such that $B \subseteq$ $A \subseteq \operatorname{cl}(B) .[13]$

An IFS $A$ is called intuitionistic fuzzy semi closed set, intuitionistic fuzzy $\alpha$-closed set, intuitionistic fuzzy preclosed set, intuitionistic fuzzy regular closed set and intuitionistic fuzzy semi-preclosed set, respectively (IFSCS, IF $\alpha$ CS, IFPCS, IFRCS and IFSPCS resp), if the complement $\bar{A}$ is an IFSOS, IF $\alpha$ OS, IFPOS, IFROS and IFSPOS respectively.

The family of all intuitionistic fuzzy semi open (resp. intuitionistic fuzzy $\alpha$-open, intuitionistic fuzzy preopen, intuitionistic fuzzy regular open and intuitionistic fuzzy semi-preopen) sets of an IFTS $(X, \tau)$ is denoted by $\operatorname{IFSO}(X)(\operatorname{resp} \operatorname{IF} \alpha(X), \operatorname{IFPO}(X), \operatorname{IFRO}(X)$ and $\operatorname{IFSPO}(X))$.

Definition 2.8 ([12]). An intuitionistic fuzzy set $A$ of an intuitionistic fuzzy topological space $(X, \tau)$ is called an intuitionistic fuzzy semi-generalized closed set (IFSGCS) if $\operatorname{scl}(A) \subseteq U$, whenever $A \subseteq U$ and $U$ is intuitionistic fuzzy semi-open set.

The complement $\bar{A}$ of intutitionistic fuzzy semi-generalized closed set $A$ is called intuitionistic fuzzy semi-generalized open set (IFSGOS).

Definition 2.9 ([12]). An intuitionistic fuzzy topological space $(X, \tau)$ is said to be intuitionistic fuzzy semi- $T_{1 / 2}$ space, if every intuitionistic fuzzy $s g$-closed set in $X$ is intuitionistic fuzzy semi- closed in $X$.

Definition 2.10 ([8]). Let $p(\alpha, \beta)$ be an IFP of an $\operatorname{IFTS}(X, \tau)$. An IFS $A$ of $X$ is called an intuitionistic fuzzy neighbourhood (IFN) of $p(\alpha, \beta)$, if there exists an IFOS $B$ in $X$ such that $p(\alpha, \beta) \in B \subseteq A$.

Definition 2.11. Let $f: X \rightarrow Y$ be a mapping from an IFTS $X$ into an IFTS $Y$. The mapping $f$ is called

(i) intuitionistic fuzzy continuous, if $f^{-1}(B)$ is an IFOS in $X$, for each IFOS $B$ in $Y$.[6] 
(ii) intuitionistic fuzzy semi-continuous, if $f^{-1}(B)$ is an IFSOS in $X$, for each IFOS $B$ in $Y$.[6]

(iii) intuitionistic fuzzy pre-continuous, if $f^{-1}(B)$ is an IFPOS in $X$, for each IFOS $B$ in $Y$.[6]

(iv) intuitionistic fuzzy $\alpha$-continuous, if $f^{-1}(B)$ is an IF $\alpha$ OS in $X$, for each IFOS $B$ in $Y$.[6]

(v) intuitionistic fuzzy semi-pre continuous, if $f^{-1}(B)$ is an IFSPOS in $X$, for each IFOS $B$ in $Y$.[13]

(vi) intuitionistic fuzzy completely continuous, if $f^{-1}(B)$ is an IFROS in $X$, for each IFOS $B$ in $Y .[15]$

Lemma 2.12 ([15]). Let $g: X \rightarrow X \times Y$ be the graph of a function $f: X \rightarrow Y$. If $A$ is an IFS of $X$ and $B$ is an IFS of $Y$, then $g^{-1}(A \times B)(x)=\left(A \cap f^{-1}(B)\right)(x)$.

\section{Intuitionistic fuzzy semi-generalized continuous mappings}

In this section we introduce intuitionistic fuzzy semi-generalized continuous mappings and studied some of the properties regarding it.

Definition 3.1. Let $A$ be an IFS in an IFTS $(X, \tau)$. Then the intuitionistic fuzzy semi-generalized interior and intuitionistic fuzzy semi-generalized closure of A are defined as follows.

$$
\begin{aligned}
\operatorname{sgint}(A) & =\cup\{G \mid G \text { is an IFSGOS in } X \text { and } G \subseteq A\}, \\
\operatorname{sgcl}(A) & =\cap\{K \mid K \text { is an IFSGCS in } X \text { and } A \subseteq K\} .
\end{aligned}
$$

Example 3.2. Let $X=\{a, b\}$.

$$
\begin{aligned}
& \text { Let } A=\left\langle x,\left(\begin{array}{cc}
\underline{a} & \underline{b} \\
0.2 & 0.3
\end{array}\right),\left(\begin{array}{cc}
\underline{a} & \underline{b} \\
0.7 & 0.7
\end{array}\right)\right\rangle \\
& B=\left\langle x,\left(\begin{array}{cc}
\underline{a}, & \underline{b} \\
0.4 & 0.7
\end{array}\right),\left(\begin{array}{cc}
\underline{a}, & \underline{b} \\
0.6 & 0.1
\end{array}\right)\right\rangle .
\end{aligned}
$$

Then $\tau=\left\{0_{\sim}, 1_{\sim}, A, B\right\}$ is an IFTS on $X$.

Then $\operatorname{sgint}(B)=A \cup 0_{\sim}=A$ and $\operatorname{sgcl}(B)=1_{\sim}$.

Proposition 3.3. If $A$ be an IFS in $X$, then $A \leq \operatorname{sgcl}(A) \leq \operatorname{scl}(A) \leq \operatorname{cl}(A)$.

Proof. The result follows from Definition.

Theorem 3.4. If $A$ is an IFSGCS in $X$, then $\operatorname{sgcl}(A)=A$.

Proof. Since $A$ is an IFSGCS, $\operatorname{sgcl}(A)$ is the smallest IFSGCS which contains $A$, which is nothing but $A$. Hence $\operatorname{sgcl}(A)=A$. 
Theorem 3.5. If $A$ is an IFSGOS in $X$, then $\operatorname{sgint}(A)=A$.

Proof. Similar to the above theorem.

Definition 3.6. Let $(X, \tau)$ and $(Y, \kappa)$ be IFT's. A mapping $f: X \rightarrow Y$ is called intuitionistic fuzzy semi-generalized continuous (intuitionistic fuzzy $s g$-continuous), if $f^{-1}(B)$ is an IFSGCS in $X$ for every IFCS $B$ in $Y$.

Theorem 3.7. Every intuitionistic fuzzy continuous mapping is an intuitionistic fuzzy sgcontinuous mapping.

Proof. Let $B$ be an IFCS in $Y$. Then by our assumption, $f^{-1}(B)$ is an IFCS in $X$. In [12], it has been proved that every intuitionistic fuzzy closed set is an intuitionistic fuzzy $s g$-closed set in $X$. Thus $f^{-1}(B)$ IFSGCS in $X$. Hence $f$ is an intuitionistic fuzzy $s g$-continuous mapping.

The following example shows that the converse of above theorem is not true in general.

Example 3.8. Let $X=\{a, b\}, Y=\{c, d\}$.

$$
\text { Let } \begin{aligned}
A & =\left\langle x,\left(\begin{array}{cc}
\underline{a}, & \underline{b} \\
0.3 & 0.4
\end{array}\right),\left(\begin{array}{cc}
\underline{a}, \underline{b} \\
0.7 & 0.6
\end{array}\right)\right\rangle \\
B & =\left\langle x,\left(\begin{array}{cc}
\underline{c}, & \underline{d} \\
0.7 & 0.8
\end{array}\right),\left(\begin{array}{cc}
\underline{c}, & \underline{d} \\
0.3 & 0.2
\end{array}\right)\right\rangle .
\end{aligned}
$$

Then $\tau=\left\{0_{\sim}, 1_{\sim}, A\right\}$ and $\kappa=\left\{0_{\sim}, 1_{\sim}, B\right\}$ are IFTS on $X$ and $Y$ respectively. Define a mapping $f:(X, \tau) \rightarrow(Y, \kappa)$ by $f(a)=c, f(b)=d$. Clearly $f$ is intuitionistic fuzzy sg-continuous map.

Now we have $f^{-1}(B)=\left\langle x,\left(\begin{array}{cc}\underline{a}, & \underline{b} \\ 0.7 & 0.8\end{array}\right),\left(\begin{array}{cc}\underline{a}, & \underline{b} \\ 0.3 & 0.2\end{array}\right)\right\rangle \cdot f^{-1}(B) \notin \tau$, which shows that $f$ is not an intuitionistic fuzzy continuous map.

Theorem 3.9. Every intuitionistic fuzzy $\alpha$-continuous mapping is an intutitionistic fuzzy sgcontinuous mapping.

Proof. Let $B$ be an IFCS in $Y$. Since $f$ is an intutitionistic fuzzy $\alpha$-continuous mapping, $f^{-1}(B)$ is an intuitionistic fuzzy $\alpha$-closed set in $X$. In [12], it has been proved that every IF $\alpha$ CS is an intuitionistic fuzzy $s g$-closed set in $X$. Thus $f^{-1}(B)$ IFSGCS in $X$. Hence $f$ is an intuitionistic fuzzy $s g$-continuous mapping.

The following example shows that the converse of the above theorem is not true in general. 
Example 3.10. Let $X=\{a, b\}, Y=\{u, v\}$.

$$
\begin{aligned}
& \text { Let } A=\left\langle x,\left(\begin{array}{cc}
\underline{a}, & \underline{b} \\
0.7 & 0.5
\end{array}\right),\left(\begin{array}{cc}
\underline{a}, & \underline{b} \\
0.3 & 0.5
\end{array}\right)\right\rangle \\
& B=\left\langle y,\left(\begin{array}{cc}
\underline{u}, & \underline{v} \\
0.25 & 0.3
\end{array}\right),\left(\begin{array}{cc}
\underline{u}, & \underline{v} \\
0.2 & 0.2
\end{array}\right)\right\rangle .
\end{aligned}
$$

Then $\tau=\left\{0_{\sim}, 1_{\sim}, A\right\}$ and $\kappa=\left\{0_{\sim}, 1_{\sim}, B\right\}$ are IFTS on $X$ and $Y$ respectively. Define a mapping $f:(X, \tau) \rightarrow(Y, \kappa)$ by $f(a)=u, f(b)=v$. Clearly $f$ is intuitionistic fuzzy sg-continuous map.

Now we have $f^{-1}(B)=\left\langle x,\left(\begin{array}{cc}\underline{a} & , \underline{b} \\ 0.25 & 0.3\end{array}\right),\left(\begin{array}{cc}\underline{a} & , \underline{b} \\ 0.2 & 0.2\end{array}\right)\right\rangle$.

$\operatorname{cl}\left(f^{-1}(B)\right)=1_{\sim}, \operatorname{int}\left(\operatorname{cl}\left(f^{-1}(B)\right)\right)=\operatorname{int}\left(1_{\sim}\right)=1_{\sim}$

$\operatorname{cl}\left(\operatorname{int}\left(\operatorname{cl}\left(f^{-1}(B)\right)\right)\right)=\operatorname{cl}\left(1_{\sim}\right)=1_{\sim}$. Thus $\operatorname{cl}\left(\operatorname{int}\left(\operatorname{cl}\left(f^{-1}(B)\right)\right)\right) \nsubseteq f^{-1}(B)$, which shows that $f$ is not an intuitionistic fuzzy $\alpha$-continuous map.

Thus the class of intuitionistic fuzzy $\alpha$-continuous maps is properly contained in the class of intutitionistic fuzzy $s g$-continuous maps.

Forthcoming theorem and example shows that the class of intuitionistic fuzzy semi-continuous maps is properly contained in the class of intuitionistic fuzzy $s g$-continuous maps.

Theorem 3.11. Every intuitionistic fuzzy semi-continuous mapping is intuitionistic fuzzy sgcontinuous mapping.

Proof. Let $f: X \rightarrow Y$ be any function from IFTS $X$ in to $Y$ such that $f$ is intuitionistic fuzzy semi-continuous. By definition of intuitionistic fuzzy semi-continuous, $f^{-1}(A)$ is IFSCS in $X$ for every IFCS $A$ in $Y$. In [12], it has been proved that every intuitionistic fuzzy semi-closed set is an intuitionistic fuzzy $s g$-closed set in $X$. Thus $f^{-1}(B)$ IFSGCS in $X$. Hence $f$ is an intuitionistic fuzzy $s g$-continuous mapping.

The converse of the above theorem is not true as seen from the following example.

Example 3.12. Let $X=\{a, b\}, Y=\{u, v\}$.

$$
\begin{aligned}
& \text { Let } A=\left\langle x,\left(\begin{array}{cc}
\underline{a}, & \underline{b} \\
0.2 & 0.4
\end{array}\right),\left(\begin{array}{cc}
\underline{a}, & \underline{b} \\
0.6 & 0.25
\end{array}\right)\right\rangle \\
& B=\left\langle y,\left(\begin{array}{cc}
\underline{u}, & \underline{v} \\
0.3 & 0.5
\end{array}\right),\left(\begin{array}{cc}
\underline{u}, \underline{v} \\
0.4 & 0.5
\end{array}\right)\right\rangle .
\end{aligned}
$$

Then $\tau=\left\{0_{\sim}, 1_{\sim}, A\right\}$ and $\kappa=\left\{0_{\sim}, 1_{\sim}, B\right\}$ are IFTS on $X$ and $Y$ respectively. Define a mapping $f: X \rightarrow Y$ by $f(a)=u, f(b)=v$. Clearly $f$ is intuitionistic fuzzy $s g$-continuous map. 
Now we have $f^{-1}(B)=\left\langle x,\left(\begin{array}{cc}\underline{a} & \underline{b} \\ 0.3 & 0.5\end{array}\right),\left(\begin{array}{cc}\underline{a}, & \underline{b} \\ 0.4 & 0.5\end{array}\right)\right\rangle$.

$\operatorname{cl}\left(f^{-1}(B)\right)=1_{\sim} . \operatorname{int}\left[\operatorname{cl}\left(f^{-1}(B)\right)\right]=\operatorname{int}\left(1_{\sim}\right)=1_{\sim}$. Thus $\operatorname{int}\left[\operatorname{cl}\left(f^{-1}(B)\right)\right] \nsubseteq f^{-1}(B)$, which shows that ' $f$ ' is not intuitionistic fuzzy semi-continuous mapping.

Theorem 3.13. Every intuitionistic fuzzy sg-continuous mapping is intuitionistic fuzzy semipre continuous mapping.

Proof. Let $B$ be an IFCS in $Y$. Since $f$ is intuitionistic fuzzy $s g$-continuous map, $f^{-1}(B)$ is an intuitionistic fuzzy $s g$-closed set in $X$. In paper [12], it has been proved that, every intuitionistic fuzzy $s g$-closed set is an intuitionistic fuzzy semi-pre closed set. Therefore $f^{-1}(B)$ is an IFSPCS in $X$. Hence $f$ is an intuitionistic fuzzy semi-pre continuous mapping.

The converse of the above theorem is not true as seen from the following example.

Example 3.14. Let $X=\{a, b\}, Y=\{u, v\}$.

$$
\begin{aligned}
& \text { Let } A=\left\langle x,\left(\begin{array}{cc}
\underline{a}, & \underline{b} \\
0.4 & 0.5
\end{array}\right),\left(\begin{array}{cc}
\underline{a}, & \underline{b} \\
0.1 & 0.3
\end{array}\right)\right\rangle \\
& B=\left\langle y,\left(\begin{array}{cc}
\underline{u}, & \underline{v} \\
0.15 & 0.3
\end{array}\right),\left(\begin{array}{cc}
\underline{u}, & \underline{v} \\
0.5 & 0.7
\end{array}\right)\right\rangle .
\end{aligned}
$$

Then $\tau=\left\{0_{\sim}, 1_{\sim}, A\right\}$ and $\kappa=\left\{0_{\sim}, 1_{\sim}, B\right\}$ are IFTS on $X$ and $Y$ respectively. Define a mapping $g:(X, \tau) \rightarrow(Y, \kappa)$ by $g(a)=u, g(b)=v$. Clearly $g$ is intuitionistic fuzzy semi-pre continuous map. Infact we have

$$
g^{-1}(B)=\left\langle x,\left(\begin{array}{cc}
\underline{a} & , \underline{b} \\
0.15 & 0.3
\end{array}\right),\left(\begin{array}{cc}
\underline{a}, \underline{b} \\
0.5 & 0.7
\end{array}\right)\right\rangle
$$

$\operatorname{scl}\left(g^{-1}(B)\right)=1 \sim \nsubseteq A$. Hence $g$ is not intuitionistic fuzzy semi-generalized continuous mapping.

Remark 3.15. Intuitionistic fuzzy pre-continuity is independent from intuitionistic fuzzy $s g$ continuity.

The proof follows from the following examples.

Example 3.16. Let $X=\{a, b\}, Y=\{u, v\}$.

$$
\begin{aligned}
& \text { Let } A=\left\langle x,\left(\begin{array}{cc}
\underline{a} & \underline{b} \\
0.3 & 0.4
\end{array}\right),\left(\begin{array}{cc}
\underline{a}, & \underline{b} \\
0.7 & 0.6
\end{array}\right)\right\rangle \\
& B=\left\langle y,\left(\begin{array}{cc}
\underline{u} & \underline{v} \\
0.6 & 0.5
\end{array}\right),\left(\begin{array}{cc}
\underline{u} & \underline{v} \\
0.4 & 0.5
\end{array}\right)\right\rangle .
\end{aligned}
$$


Then $\tau=\left\{0_{\sim}, 1_{\sim}, A\right\}$ and $\kappa=\left\{0_{\sim}, 1_{\sim}, B\right\}$ are IFTS on $X$ and $Y$ respectively. Define a mapping $f:(X, \tau) \rightarrow(Y, \kappa)$ by $f(a)=u, f(b)=v$. Clearly $f$ is intuitionistic fuzzy $s g$-continuous map. Infact we have

$$
f^{-1}(B)\left\langle x,\left(\begin{array}{cc}
\underline{a} & , \underline{b} \\
0.15 & 0.3
\end{array}\right),\left(\begin{array}{cc}
\underline{a}, & \underline{b} \\
0.5 & 0.7
\end{array}\right)\right\rangle
$$

$\left(\operatorname{cl}\left(f^{-1}(B)\right)=1 \sim \cap \bar{A}=\bar{A} \cdot \operatorname{int}\left[\operatorname{cl}\left(f^{-1}(B)\right)\right]=\operatorname{int}(\bar{A})=0 \sim \cup A=A\right.$. Hence $f^{-1}(B) \nsubseteq A=\operatorname{int}\left[\operatorname{cl}\left(f^{-1}(B)\right)\right]$ which shows that $f$ is not an intuitionistic fuzzy pre-continuous map.

Example 3.17. Let $X=\{a, b\}, Y=\{u, v\}$.

$$
\begin{aligned}
& \text { Let } A=\left\langle x,\left(\begin{array}{cc}
\underline{a}, & \underline{b} \\
0.4 & 0.5
\end{array}\right),\left(\begin{array}{cc}
\underline{a}, & \underline{b} \\
0.5 & 0.6
\end{array}\right)\right\rangle \\
& B=\left\langle y,\left(\begin{array}{cc}
\underline{u} & \underline{v} \\
0.2 & 0.3
\end{array}\right),\left(\begin{array}{cc}
\underline{u} & \underline{v} \\
0.4 & 0.7
\end{array}\right)\right\rangle .
\end{aligned}
$$

Then $\tau=\left\{0_{\sim}, 1_{\sim}, A\right\}$ and $\kappa=\left\{0_{\sim}, 1_{\sim}, B\right\}$ are IFTS on $X$ and $Y$ respectively. Define a mapping $h:(X, \tau) \rightarrow(Y, \kappa)$ by $h(a)=u, h(b)=v$. Clearly $h$ is intuitionistic fuzzy pre-continuous map. Infact we have

$$
h^{-1}(B)=\left\langle x,\left(\begin{array}{cc}
\underline{a} & , \underline{b} \\
0.2 & 0.3
\end{array}\right),\left(\begin{array}{cc}
\underline{a} & , \underline{b} \\
0.4 & 0.7
\end{array}\right)\right\rangle
$$

$\operatorname{scl}\left(h^{-1}(B)\right)=1 \sim h^{-1}(B) \subset A$, but $\operatorname{scl}\left(h^{-1}(B)\right) \not \subset A$, which shows that $f$ is not an intuitionistic fuzzy $s g$-continuous map.

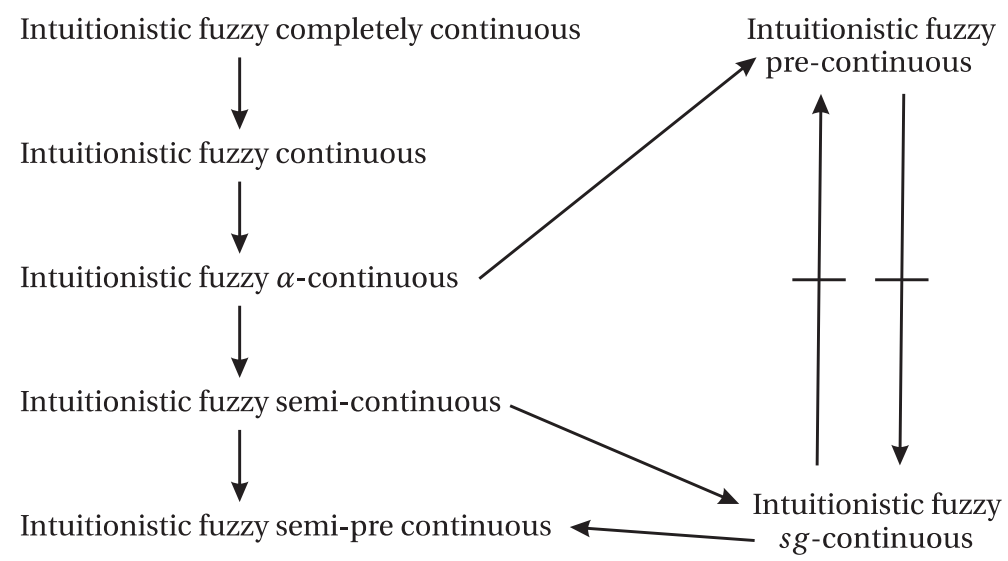

The above diagram shows the relationships between intuitionistic fuzzy $s g$-continuous mappings and some other mappings. The reverse implications are not true in the above diagram. 
Theorem 3.18. Let $f: X \rightarrow Y$ be a mapping from a IFTS $X$ into an IFTS $Y$. Then the following statements are equivalent.

(i) $f$ is intuitionistic fuzzy sg-continuous mapping.

(ii) $f^{-1}(B)$ is an IFSGOS in $X$, for every IFOS B in $X$.

Proof. (i) $\Rightarrow$ (ii) Let $B$ be an IFOS in $Y$, then $\bar{B}$ is an IFCS in $Y$. Since $f$ is intuitionistic fuzzy $s g$-continuous mapping $f^{-1}(\bar{B})$ is an IFSGCS in $X$. Then $f^{-1}(\bar{B})=\overline{f^{-1}(B)}$, implies $f^{-1}(B)$ is an IFSGOS in $Y$.

(ii) $\Rightarrow$ (i) Let $B$ be an IFCS in $Y$. By our assumption $f^{-1}(\bar{B})$ is an IFSGOS in $X$ for every IFOS $\bar{B}$ in $Y$. But $f^{-1}(\bar{B})=\overline{f^{-1}(B)}$, which in turn implies $f^{-1}(B)$ is an IFSGCS in $X$. Hence $f$ is intuitionistic fuzzy $s g$-continuous mapping.

Theorem 3.19. Let $f: X \rightarrow Y$ be an intuitionistic fuzzy sg-continuous mapping. Then the following statements hold.

(i) $f(\operatorname{sgcl}(A)) \leq c 1(f(A))$, for every intuitionistic fuzzy set $A$ in $X$.

(ii) $\operatorname{sgcl}\left(f^{-1}(B)\right) \leq f^{-1}(c 1(B))$ for every intuitionistic fuzzy set $B$ in $Y$.

Proof. (i) Let $A \leq X$. Then $\operatorname{cl}(f(A))$ is an intuitionistic fuzzy closed set in $Y$. Since $f$ is intuitionistic fuzzy $s g$-continuous, $f^{-1}[\mathrm{c} 1[f(A)]]$ is intuitionistic fuzzy $s g$-closed in $X$. Since $A \leq f^{-1}(f(A)) \leq f^{-1}[\mathrm{c} 1[f(A)]]$ and $f^{-1}[\mathrm{c} 1[f(A)]]$ is intuitionistic fuzzy sg-closed, implies $\operatorname{sgcl}(A) \leq f^{-1}[\operatorname{cl}(f(A))]$. Hence $f[\operatorname{sgcl}(A)] \leq \operatorname{cl}[f(A)]$.

(ii) Replacing $A$ by $f^{-1}(B)$ in (i), we get

$$
\begin{aligned}
& f\left[\operatorname{sgcl}\left(f^{-1}(B)\right)\right] \leq \operatorname{cl}\left[f\left(f^{-1}(B)\right)\right]=\operatorname{cl}(B) \\
& f\left[\operatorname{sgcl}\left(f^{-1}(B)\right)\right] \leq \operatorname{cl}(B)
\end{aligned}
$$

Hence $\operatorname{sgc} 1\left[f^{-1}(B)\right] \leq f^{-1}[\mathrm{c} 1(B)]$.

Theorem 3.20. Let $f: X \rightarrow Y$ be a function and $g: X \rightarrow X \times Y$ the graph of the function $f$. Then $f$ is intuitionistic fuzzy sg-continuous if $g$ is so.

Proof. Let $B$ be an IFOS in $Y$. Then by Lemma 2.11, $f^{-1}(B)=f^{-1}\left(1_{\sim} \times B\right)=1 \sim \cap f^{-1}(B)=$ $g^{-1}\left(1_{\sim} \times B\right)$. Since $B$ is an IFOS $Y, 1_{\sim} \times B$ is an IFOS in $X \times Y$. Also since $g$ is intuitionistic fuzzy $s g$-continuous implies that $g^{-1}\left(1_{\sim} \times B\right)$ is an IFSGOS in $X$. Therefore $f^{-1}(B)$ is an IFSGOS in $X$. Hence $f$ is intuitionistic fuzzy $s g$-continuous mapping.

Theorem 3.21. Let $f: X \rightarrow Y$ is a mapping from an IFTS $X$ into an IFTS $Y$. If any union of IFSGCS is IFSGCS, then the following statements are equivalent. 
(i) $f$ is intuitionistic fuzzy sg-continuous mapping.

(ii) For each IFP $p_{(\alpha, \beta)} \in X$ and every IFN A of $f\left(p_{(\alpha, \beta)}\right)$, there exists an IFSGCS B such that $p_{(\alpha, \beta)} \in B \leq f^{-1}(A)$.

(iii) For each IFP $p_{(\alpha, \beta)} \in X$ and every IFN $A$ of $f\left(p_{(\alpha, \beta)}\right)$, there exists an IFSGCS B such that $p_{(\alpha, \beta)} \in B$ and $f(B) \leq A$.

Proof. (i) $\Rightarrow$ (ii): Assume that $f$ is intuitionistic fuzzy $s g$-continuous. Let $p_{(\alpha, \beta)}$ be an IFP in $X$ and $A$ be an IFN of $f\left(p_{(\alpha, \beta)}\right)$. Then by Definition of IFN, there exists an IFCS $C$ in $Y$, such that $f\left(p_{(\alpha, \beta)}\right) \in C \leq A$. Taking $B=f^{-1}(C) \in X$, since $f$ is intuitionistic fuzzy $s g$-continuous, $f^{-1}(C)$ is IFSGCS and

$$
p_{(\alpha, \beta)} \in B \leq f^{-1}\left[f\left(p_{(\alpha, \beta)}\right)\right] \leq f^{-1}(C)=B \leq f^{-1}(A) .
$$

Hence $p_{(\alpha, \beta)} \in B \leq f^{-1}(A)$.

(ii) $\Rightarrow$ (iii): Let $p_{(\alpha, \beta)}$ be an IFP in $X$ and $A$ be an IFN of $f\left(p_{(\alpha, \beta)}\right)$, such that there exists an IFSGCS $B$ with $p_{(\alpha, \beta)} \in B \leq f^{-1}(A)$. From this we have $p_{(\alpha, \beta)} \in B$ and $B \leq f^{-1}(A)$. This implies $f(B) \leq f\left(f^{-1}(A)\right)=A$. Hence (iii) holds.

(iii) $\Rightarrow$ (i): Assume that (iii) holds. Let $B$ be an IFCS in $Y$ and take $p_{(\alpha, \beta)} \in f^{-1}(B)$. Then $f\left(p_{(\alpha, \beta)}\right) \in f\left(f^{-1}(B)\right) \leq B$. Since $B$ is IFCS in $Y$, it follows that $B$ is an IFN of $f\left(p_{(\alpha, \beta)}\right)$. Then from (iii), there exists an IFSGCS $A$ such that $p_{(\alpha, \beta)} \in A$ and $f(A) \leq B$. This shows that $p_{(\alpha, \beta)} \in$ $A \leq f^{-1}(f(A)) \leq f^{-1}(B)$. (i.e) $p_{(\alpha, \beta)} \in A \subseteq f^{-1}(B)$. Since $p(\alpha, \beta)$ is an arbitrary IFP and $f^{-1}(B)$ is union of all IFP contained in $f^{-1}(B)$, by assumption $f^{-1}(B)$ is an IFSGCS. Hence $f$ is intuitionistic fuzzy $s g$-continuous mapping.

Theorem 3.22. Let $f: X \rightarrow Y$ is a mapping from an IFTS $X$ into an IFTS $Y$. Then the following statements are equivalent.

(i) $f$ is intuitionistic fuzzy sg-continuous mapping.

(ii) $f^{-1}(B)$ is an IFSGOS in $X$, for every IFOS B in $X$.

(iii) $f(\operatorname{sgcl}(A)) \leq c 1(f(A))$, for every fuzzy set $A$ in $X$.

(iv) $\operatorname{sgcl}\left(f^{-1}(B)\right) \leq f^{-1}(c 1(B))$ for every fuzzy set $B$ in $Y$.

(v) For each IFP $p_{(\alpha, \beta)} \in X$ and every IFN A of $f\left(p_{(\alpha, \beta)}\right)$, there exists an IFSGCS B such that $p_{(\alpha, \beta)} \in B \leq f^{-1}(A)$.

(vi) For each IFP $p_{(\alpha, \beta)} \in X$ and every IFN A of $f\left(p_{(\alpha, \beta)}\right)$, there exists an IFSGCS B such that $p_{(\alpha, \beta)} \in B$ and $f(B) \leq A$.

Proof. Follows form the Theorems.3.18, 3.19 and 3.22.

Theorem 3.23. If $f: X \rightarrow Y$ is intuitionistic fuzzy sg-continuous and $g: Y \rightarrow Z$ is intuitionistic fuzzy completely continuous, then $g \circ f: X \rightarrow Z$ is intuitionistic fuzzy sg-continuous. 
Proof. Let $B$ be any IFCS in $Z$. Since $g$ is intuitionistic fuzzy completely continuous, $g^{-1}(B)$ is an IFRCS in $Y$. In [6], it has been proved that every IFRCS is an IFCS. Therefore $g^{-1}(B)$ is an IFCS in $Y$. Also since $f$ is intuitionistic fuzzy $s g$-continuous mapping $f^{-1}\left[g^{-1}(B)\right]$ is an IFSGCS in $X$.

We have $(g \circ f)^{-1}[B]=f^{-1}\left[g^{-1}(B)\right]$ is IFSGCS in $X$, for every IFCS $B$ in $Z$. Hence $g \circ f$ is intuitionistic fuzzy $s g$-continuous mapping.

Theorem 3.24. If $: X \rightarrow Y$ is intuitionistic fuzzysg-continuous and $g: Y \rightarrow Z$ is intuitionistic fuzzy continuous, then $g \circ f: X \rightarrow Z$ is intuitionistic fuzzy sg-continuous.

Proof. Let $B$ be any intuitionistic fuzzy closed set in $Z$. Since $g$ is intuitionistic fuzzy continuous, $g^{-1}(B)$ is intuitionistic fuzzy closed set in $Y$. Since $f$ is intuitionistic fuzzy $s g$-continuous mapping $f^{-1}\left[g^{-1}(B)\right]$ is an intuitionistic fuzzy $s g$-closed set in $X$.

$(g \circ f)^{-1}[B]=f^{-1}\left[g^{-1}(B)\right]$ is intuitionistic fuzzy $s g$-closed set, for every intuitionistic fuzzy closed $B$ in $Z$.

Hence $g \circ f$ is intuitionistic fuzzy $s g$-continuous mapping.

Theorem 3.25. Let $f: X \rightarrow Y$ is a mapping from an IFTS $X$ into an IFTS $Y$. If $X$ is intuitionistic fuzzy semi-T $T_{1 / 2}$ space, then $f$ is intuitionistic fuzzy sg-continuous iff it is intuitionistic fuzzy semi-continuous.

Proof. Let $f$ be intuitionistic fuzzy $s g$-continuous mapping and let $A$ be an intuitionistic fuzzy closed set in $Y$. Then by definition of intuitionistic fuzzy semi-generalized continuous $f^{-1}(A)$ is intuitionistic fuzzy $s g$-closed in $X$. Since $X$ is intuitionistic fuzzy semi- $T_{1 / 2}$ space, $f^{-1}(A)$ is intuitionistic fuzzy semi-closed set.

Hence $f$ is intuitionistic fuzzy semi-continuous.

Conversely assume that $f$ is intuitionistic fuzzy semi-continuous. Then by Theorem 3.11 $f$ is intuitionistic fuzzy $s g$-continuous mapping.

Theorem 3.26. Let $X, X_{1}, X_{2}$ are IFTS's and $p_{i}: X_{1} \times X_{2} \rightarrow X_{i}(i=1,2)$ are projections of $X_{1} \times$ $X_{2}$ onto $X_{i}$. If $f: X \rightarrow X_{1} \times X_{2}$ is intuitionistic fuzzy sg-continuous, then $p_{i} \circ f(i=1,2)$ is intuitionistic fuzzy sg-continuous mapping.

Proof. It follows from the facts that projections are intuitionistic fuzzy continuous mappings. 


\section{Intuitionistic fuzzy semi-generalized irresolute mappings}

Definition 4.1. A mapping $f: X \rightarrow Y$ from an IFTS $X$ into an IFTS $Y$ is said to be intuitionistic fuzzy semi-generalized irresolute (intuitionistic fuzzy $s g$-irresolute) if $f^{-1}(B)$ is an IFSGCS in $X$ for every IFSGCS $B$ in $Y$.

Theorem 4.2. Let $f: X \rightarrow Y$ is a mapping from an IFTS $X$ into an IFTS $Y$. Then every intuitionistic fuzzy sg-irresolute mapping is intuitionistic fuzzy sg-continuous.

Proof. Assume that $f: X \rightarrow Y$ is an intuitionistic fuzzy $s g$-irresolute mapping and let $A$ be an IFCS in $Y$. In [12], it has been proved that every intuitionistic fuzzy closed set is an intuitionistic fuzzy $s g$-closed. Therefore $A$ is an IFSGCS in $Y$. Since $f$ is intuitionistic fuzzy $s g$-irresolute, by definition $f^{-1}(A)$ is IFSGCS in $X$. Hence $f$ is intuitionistic fuzzy $s g$-continuous.

Example 4.3. Let $X=\{a, b, c\}, Y=\{u, v, w\}$.

$$
\begin{aligned}
& \text { Let } A=\left\langle x,\left(\begin{array}{ccc}
\underline{a}, \underline{b} & \underline{c} \\
0.8 & 0.4 & 0.4
\end{array}\right),\left(\begin{array}{ccc}
\underline{a}, \underline{b} & , \underline{c} \\
0.1 & 0.6 & 0.6
\end{array}\right)\right\rangle
\end{aligned}
$$

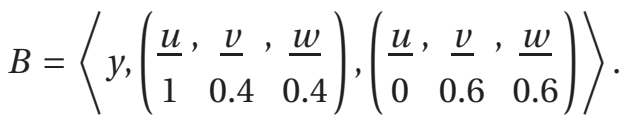

Then $\tau=\left\{0_{\sim}, 1_{\sim}, A\right\}$ and $\kappa=\left\{0_{\sim}, 1_{\sim}, B\right\}$ are IFTS on $X$ and $Y$ respectively. Define a mapping $h$ : $(X, \tau) \rightarrow(Y, \kappa)$ by $h(a)=u, h(b)=v, h(c)=w$. Clearly $h$ is intuitionistic fuzzy $s g$-continuous map. Infact we have

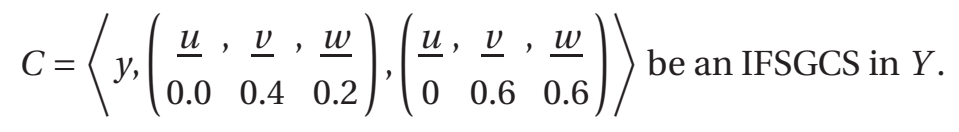

$h^{-1}(C)=\left\langle x,\left(\begin{array}{ccc}\underline{a}, \underline{b} & \underline{c} \\ 0.0 & 0.4 & 0.2\end{array}\right),\left(\begin{array}{ccc}\underline{a}, \underline{b} & \underline{c} \\ 0.0 & 0.6 & 0.6\end{array}\right)\right\rangle$.

$\operatorname{scl}\left(h^{-1}(C)\right)=1_{\sim}=1_{\sim} . h^{-1}(C) \subset A$, but $\operatorname{scl}\left(h^{-1}(C)\right) \nsubseteq A$, which shows that $h^{-1}(C)$ is not an IFSGCS in $X$. Therefore $f$ is not an intuitionistic fuzzy $s g$-irresolute map.

Theorem 4.4. Let $f: X \rightarrow Y$ be a mapping from a IFTS $X$ into an IFTS $Y$. Then the following statements are equivalent.

(i) $f$ is intuitionistic fuzzy sg-irresolute mapping.

(ii) $f^{-1}(B)$ is an IFSGOS in $X$, for every IFSGOS B in $X$.

Proof. Similar to Theorem 3.18.

Theorem 4.5. Let $f: X \rightarrow Y$ be a mapping from an IFTS $X$ into an IFTS $Y$. Then the following statements are equivalent. 
(i) $f$ is an intuitionistic fuzzy semi-generalized irresolute mapping.

(ii) $f^{-1}(B)$ is an IFSGOS in $X$ for each IFSGOS B in $Y$.

(iii) $\operatorname{sgcl}\left(f^{-1}(B)\right) \leq f^{-1}(\operatorname{sgcl}(B))$, for each IFS B of $Y$.

(iv) $f^{-1}(\operatorname{sgint} B) \leq \operatorname{sgint}\left[f^{-1}(B)\right]$, for each IFS B of $Y$.

Proof. (i) $\Rightarrow$ (ii) It can be proved by using the complement and Definition 4.1.

(ii) $\Rightarrow$ (iii) Let $B$ be an IFS in $Y$. Since $B \leq \operatorname{sgcl}(B), f^{-1}(B)=f^{-1}(\operatorname{sgcl}(B))$. Since $\operatorname{sgcl}(B)$ is an IFSGCS in $Y$, by our assumption, $f^{-1}(\operatorname{sgcl}(B))$ is an IFSGCS in $X$. Therefore $\operatorname{sgcl}\left[f^{-1}(B)\right] \leq$ $f^{-1}(\operatorname{sgcl}(B))$.

(iii) $\Rightarrow$ (iv) By taking complement we get the result.

(iv) $\Rightarrow$ (i) Let $B$ be any IFSGOS in $Y$. Then $\operatorname{sgint}(B)=B$. By our assumption we have $f^{-1}(B)=$ $f^{-1}(\operatorname{sgint}(B)) \leq \operatorname{sgint}\left[f^{-1}(B)\right]$, so $f^{-1}(B)$ is an IFSGOS in $X$. Hence $f$ is intuitionistic fuzzy $s g$-irresolute mapping.

Theorem 4.6. Let $f: X \rightarrow Y$ be intuitionistic fuzzy sg-irresolute mapping. Then $f$ is intuitionistic fuzzy irresolute mapping if $(X, \tau)$ is intuitionistic fuzzy semi-T $T_{1 / 2}$ space.

Proof. Let $B$ be an IFSCS in $Y$. Then $B$ is an IFSGCS in $Y$. Since $f$ is intuitionistic fuzzy $s g$ irresolute, $f^{-1}(B)$ is an IFSGCS in $X$. But $(X, \tau)$ is intuitionistic fuzzy semi- $T_{1 / 2}$ space implies $f^{-1}(B)$ is an IFSCS in $X$. Hence $f$ is intuitionistic fuzzy irresolute.

Theorem 4.7. If a mapping $f: X \rightarrow Y$ is intuitionistic fuzzy sg-irresolute mapping, then $f(\operatorname{sgcl}(B)) \leq \operatorname{scl}(f(B))$ for every IFS $B$ of $X$.

Proof. Let $B$ be an IFS of $X$. Since $\operatorname{scl}(f(B))$ is an IFSGCS in $Y$, by our assumption $f^{-1}$ [scl $(f(B))]$ is an IFSGCS in $X$. Furthermore $B \leq f^{-1}(f(B)) \leq f^{-1}(\operatorname{scl}(f(B)))$ and hence $\operatorname{sgcl}(B) \leq$ $f^{-1}[\operatorname{scl}(f(B))]$ and consequently $f[\operatorname{sgcl}(B)] \leq f\left[f^{-1}[\operatorname{scl}(f(B))]\right] \leq \operatorname{scl}(f(B))$.

Theorem 4.8. Let $(Y, \kappa)$ be an IFTS such that every IFSCS in $Y$ is an IFCS. If $f:(X, \tau) \rightarrow(Y, \kappa)$ is bijective and intuitionistic fuzzy sg-continuous then $f$ is intuitionistic fuzzy sg-irresolute.

Proof. Let $B$ be an IFSGCS in $Y$ and let $f^{-1}(B) \leq A$, where $A$ is an IFSOS in $X$. Then $B \leq$ $f(A)$. Since $f(A)$ is an IFSOS in $Y$ and $B$ is an IFSGCS in $Y$, then $\operatorname{scl}(B) \leq f(A)$ and hence $f^{-1}(\operatorname{scl}(A)) \leq f^{-1}(f(A))=A$. Since $f$ is intuitionistic fuzzy $s g$-continuous and $\operatorname{scl}(B)$ is an IFCS in $Y$, then $f^{-1}(\operatorname{scl}(B))$ is an IFSGCS in $X$. Therefore $\operatorname{scl}\left[f^{-1}(\operatorname{scl}(B))\right] \leq A$ and $\operatorname{soscl}\left(f^{-1}(B)\right)$ $\leq A$. Hence $f^{-1}(B)$ is an IFSGCS in $X$. Hence $f$ is intuitionistic fuzzy $s g$-irresolute mapping. 
Theorem 4.9. Let $f: X \rightarrow Y$ be an intuitionistic fuzzy sg-irresolute mappings. Then $f$ is intuitionistic fuzzy irresolute, if $(X, \tau)$ is an intuitionistic fuzzy semi- $T_{1 / 2}$ space.

Proof. Let $A$ be any IFSCS in $Y$. In [12], it has been proved that every IFSCS is an IFSGCS. Therefore $A$ is an IFSGCS in $Y$ and $f$ is an intuitionistic fuzzy $s g$-irresolute. Then by definition $f^{-1}(A)$ is IFSGCS in $X$. But $(X, \tau)$ is an intuitionistic fuzzy semi- $T_{1 / 2}$ space, so $f^{-1}(A)$ is an IFSCS. Hence $f$ is an intuitionistic fuzzy irresolute.

Theorem 4.10. If any union of IFSGCS is an IFSGCS, then a mapping $f: X \rightarrow Y$ from an IFTS $X$ into an IFTS $Y$ is intuitionistic fuzzy sg-irresolute if and only if for each IFP $p_{(\alpha, \beta)}$ in $X$ and IFSGCS B in $Y$ such that $f\left(p_{(\alpha, \beta)}\right) \in B$, there exists an IFSGCS $A$ in $X$ such that $p_{(\alpha, \beta)} \in A$ and $f(A) \leq B$.

Proof. Let $f$ be any intuitionistic fuzzy $s g$-irresolute mapping, $p_{(\alpha, \beta)}$ an IFP in $X$ and $B$ be any IFSGCS in $Y$, such that $f\left(p_{(\alpha, \beta)}\right) \in B$. Then $p_{(\alpha, \beta)} \in f^{-1}(B)=\operatorname{sgcl}\left[f^{-1}(B)\right]$. We take $A=\operatorname{sgcl}\left[f^{-1}(B)\right]$. Then $A$ is an IFSGCS in $X$, containing IFP $p_{(\alpha, \beta)}$ and $f(A)=f\left[\operatorname{sgcl}\left(f^{-1}(B)\right)\right] \leq$ $f\left[f^{-1}(B)\right] \leq B$.

Conversely assume that $B$ be any IFSGCS in $Y$ and IFP $p_{(\alpha, \beta)}$ in $X$, such that $p_{(\alpha, \beta)} \in$ $f^{-1}(B)$. By assumption there exists IFSGCS $A$ in $X$ such that $p_{(\alpha, \beta)} \in A$ and $f(A) \leq B$. Therefore $p_{(\alpha, \beta)} \in A \leq f^{-1}(B)$ and $p_{(\alpha, \beta)} \in A=\operatorname{sgcl}(A) \leq \operatorname{sgcl}\left[f^{-1}(B)\right]$. Since $p_{(\alpha, \beta)}$ is an arbitrary IFP and $f^{-1}(B)$ is union of all IFP contained in $f^{-1}(B), f^{-1}(B)$ is an IFSGCS in $X$, so $f$ is an intuitionistic fuzzy semi-generalized irresolute mapping.

Corollary 4.11. A mapping $f: X \rightarrow Y$ from an IFTS $X$ into an IFTS $Y$ is intuitionistic fuzzy semi-generalized irresolute if and only if for each IFP $p_{(\alpha, \beta)}$ in X and IFSGCS B in $Y$ such that $f\left(p_{(\alpha, \beta)}\right) \in B$, there exists an IFSGCS $A$ in $X$ such that $p_{(\alpha, \beta)} \in A$ and $A \leq f^{-1}(B)$.

Proof. Follows from Theorem 4.10.

Theorem 4.12. Let $f: X \rightarrow Y$ and $g: Y \rightarrow Z$ are intuitionistic fuzzy sg-irresolute mappings, where $X, Y, Z$ are IFTS. Then $g \circ f$ is an intuitionistic fuzzy sg-irresolute mapping.

Proof. Let $A$ be an intuitionistic fuzzy $s g$-closed set in $Z$. Since $g$ is an intuitionistic fuzzy semi-generalized irresolute mapping $g^{-1}(A)$ is an intuitionistic fuzzy $s g$-closed set in $Y$. Also since $f$ is intuitionistic fuzzy semi-generalized irresolute mapping, $f^{-1}\left[g^{-1}(A)\right]$ is an intuitionistic fuzzy $s g$-closed set in $X$.

$(g \circ f)^{-1}(A)=f^{-1}\left[g^{-1}(A)\right]$ for each $A$ in $Z$. Hence $(g \circ f)^{-1}(A)$ is an intuitionistic fuzzy $s g$-closed set in $X$. Therefore $g \circ f$ is an intuitionistic fuzzy semi-generalized irresolute mapping. 
Theorem 4.13. Let $f: X \rightarrow Y$ and $g: Y \rightarrow Z$ are intuitionistic fuzzy semi-generalized irresolute and intuitionistic fuzzy continuous mappings respectively, where $X, Y, Z$ are IFTS. Then $g \circ f$ is an intuitionistic fuzzy semi-generalized continuous mapping.

Proof. Let $A$ be any IFCS in $Z$. Since $g$ is intuitionistic fuzzy semi-generalized continuous, $g^{-1}(A)$ is an IFSGCS in $Y$. Also, since $f$ is intuitionistic fuzzy semi-generalized irresolute, $f^{-1}\left[g^{-1}(A)\right]$ is an IFSGCS in $X$.

$(g \circ f)^{-1}(A)=f^{-1}\left[g^{-1}(A)\right]$ is an IFSGCS in $X$. Hence $g \circ f$ is intuitionistic fuzzy semigeneralized continuous.

Theorem 4.14. Let $(X, \tau),(Y, \kappa),(Z, \delta)$ be any intuitionistic fuzzy topological spaces. Let $f$ : $(X, \tau) \rightarrow(Y, \kappa)$ be intuitionistic fuzzy semi-generalized irresolute and $g:(Y, \kappa) \rightarrow(Z, \sigma)$ is intuitionistic fuzzy continuous, then $g \circ f$ is intuitionistic fuzzy semi-generalized continuous.

Proof. Let $B$ be any intuitionistic fuzzy closed set in $Z$. Since $g$ is intuitionistic fuzzy continuous, $g^{-1}(B)$ is IFCS in $Y$. In paper [12], it has been proved that every IFCS is an IFSGCS. Therefore $f^{-1}(B)$ is an IFSGCS in $Y$. But since $f$ is an intuitionistic fuzzy sgirresolute mapping $f^{-1}\left(g^{-1}(B)\right)$ is an IFSGCS in $X$.

$[g \circ f]^{-1}(B)=f^{-1}\left(g^{-1}(B)\right)$ is IFSGCS in $X$ for every IFCS ' $B$ ' in $X$.

Hence $g \circ f$ is intuitionistic fuzzy $s g$-continuous.

Theorem 4.15. Let $X, X_{1}, X_{2}$ are IFTS's and $p_{i}: X_{1} \times X_{2} \rightarrow X_{i}(i=1,2)$ are projections of $X_{1} \times$ $X_{2}$ onto $X_{i}$. If $f: X \rightarrow X_{1} \times X_{2}$ is intuitionistic fuzzy semi-generalized irresolute, then $p_{i} f$ is intuitionistic fuzzy semi-generalized continuous mapping.

Proof. $p_{i} f: X \rightarrow X_{i}(i=1,2)$. It follows from the fact that $p_{i}(i=1,2)$ are intuitionistic fuzzy continuous mappings and by Theorem $\mathbf{5 . 5}$.

\section{References}

[1] K. T. Atanassov, Intuitionistic fuzzy sets, Fuzzy Sets and Systems, 20 (1986), 87-96.

[2] Biljana Krsteska and Salah Abbas, Intuitionistic fuzzy strongly irresolute precontinuous mappings in Coker's spaces, Kragujevac. J. Math., 30 (2007), 243-252.

[3] C. L. Chang, Fuzzy topological spaces, J. Math. Anal. Appl., 24 (1986), 182-190.

[4] D. Coker, An introduction to intuitionistic fuzzy topological spaces, Fuzzy Sets and Systems, 88 (1997), 81-89.

[5] M. E. El-Shafei and A. Zakai, Semi-generalized continuous mappings in fuzzy topological spaces, J. Egypt. Math. Soc., 15(2007), 57-67.

[6] H. Gurcay, D. Coker, On fuzzy continuity in intuitionistic fuzzy topological spaces, J. Fuzzy. Math., 5(1997), 365-378.

[7] J. K. Joen, Y. B. Jun and J. H. Park, Intuitionistic fuzzy alpha-continuity and intuitionistic fuzzy pre-continuity, IJMMS, 19(2005), 3091-3101. 
[8] S. J. Lee and E. P. Lee, The category of intuitionistic fuzzy topological spaces, Bull. Korean. Math. Soc., 37(2000), $63-71$.

[9] S. S. Thakur and Surendra Singh, On fuzzy semi-preopen sets and fuzzy semiprecontinuity, Fuzzy Sets and Systems, 98(1998), 383-391.

[10] S. S. Thakur and Rekka Chaturvedi, RG-Closed sets in intuitionistic fuzzy topological spaces, Universitatea Din Bacau Studii Si Cercertari Stiintifice, Nr. 16 (2006), 257-272.

[11] S. S. Thakur and Rekka Chaturvedi, Generalized continuity in intuitionistic fuzzy topological spaces. (Submitted).

[12] R. Santhi and K. Arun Prakash, Intuitionistic fuzzy semi-generalized closed sets (Submitted to Korean Mathematical Society).

[13] Young Bae Jun and Seok-Zun Song, Intuitionistic fuzzy semi-preopen sets and intuitionistic fuzzy semiprecontinuous mappings, J. Appl. Math \& Computing, 19(2005), 467-474.

[14] L. A. Zadeh, Fuzzy sets, Information Control, 8 (1965), 338-353.

[15] I. M. Hanafy, Completely continuous functions in Intuitionistic fuzzy topological spaces, Czechoslovak Mathematical Journal, 53(2003), 793-803.

Department of Mathematics, Nallamuthu Gounder Mahalingam College Pollachi - 642001.

E-mail: santhifuzzy@yahoo.co.in

Department of Mathematics, Kongu Engineering College Perundurai - 638052.

E-mail: arun.kannusamy@yahoo.co.in 